\title{
Philosophiques
}

\section{Yvon Gauthier, Hegel : introduction à une lecture critique, Québec, Presses de l’Université Laval, coll. « Logique de la science ", 2010, 105 p.}

\section{Pierre-Alexandre Fradet}

Volume 38, numéro 2, automne 2011

URI : https://id.erudit.org/iderudit/1007473ar

DOI : https://doi.org/10.7202/1007473ar

Aller au sommaire du numéro

Éditeur(s)

Société de philosophie du Québec

\section{ISSN}

0316-2923 (imprimé)

1492-1391 (numérique)

Découvrir la revue

Citer ce compte rendu

Fradet, P.-A. (2011). Compte rendu de [Yvon Gauthier, Hegel : introduction à une lecture critique, Québec, Presses de l’Université Laval, coll. « Logique de la science », 2010, 105 p.] Philosophiques, 38(2), 627-631.

https://doi.org/10.7202/1007473ar d'utilisation que vous pouvez consulter en ligne.

https://apropos.erudit.org/fr/usagers/politique-dutilisation/ 
Yvon Gauthier, Hegel: introduction à une lecture critique, Québec, Presses de l'Université Laval, coll. «Logique de la science», 2010, 105 p.

Si ce n'était du présent volume, il faudrait remonter à I 969 pour dater un ouvrage d'Yvon Gauthier qui porte spécifiquement sur la pensée hégélienne. Plus de quarante années se sont donc écoulées depuis la parution de L'arc et le cercle: l'essence du langage chez Hegel et Hölderlin ${ }^{1}$. Entre ce premier ouvrage et son plus récent, Hegel: introduction à une lecture critique, Gauthier n'a pas contribué aux études hégéliennes en se contentant de traduire le concept d'Aufhebung par celui de "sursomption", traduction qui fut adoptée par G. Jarczyk et P.-J. Labarrière ${ }^{2}$; il a aussi publié différents articles et chapitres de livre qui traitent directement de $\mathrm{Hegel}^{3}$. Le travail qu'il nous offre aujourd'hui ne manque pas de qualités: synthétique, ordonné et fluide, il fournit un bilan utile des réflexions de l'A. sur la logique dialectique de Hegel. En tenant compte de travaux passés mais aussi de commentaires plus frais (R. Brandom, R. Pippin, J. Reid, M. Robitaille, D. Perinetti et M.-A. Ricard, T. De Koninck et G. Planty-Bonjour, etc.), Gauthier se propose d'introduire à Hegel, au contraire de certains interprètes $^{4}$, en «adopt[ant] l'attitude du lecteur critique qui n'est peut-être pas toujours fidèle à l'esprit [mais reste] attentif à la lettre» (p. XII).

La lecture de Gauthier cherche à vrai dire à mettre «l'accent sur le langage de Hegel, son vocabulaire et sa syntaxe, plutôt que sa sémantique qui a une visée idéaliste et qu['il] veu[t] détourner au profit d'une critique constructive $[. .$.$] de l'échafaudage métaphysique» (p. XII). Deux objectifs$ précis sont poursuivis en l'occurrence: d'une part, «dégager sous la phénoménologie de l'esprit une phénoménologie du langage » et, d'autre part, tirer au clair "sous la science de la logique une logique interne du langage» (p. XII). Alors que la Phénoménologie de l'esprit est étudiée pour elle-même

1. Voir L'arc et le cercle: l'essence du langage chez Hegel et Hölderlin, Bruxelles-Paris et Montréal, Desclée de Brouwer et Bellarmin, I969.

2. G. W. F. Hegel, Phénoménologie de l'esprit, traduction de G. Jarczyk et P.-J. Labarrière, Paris, Gallimard, I993. Gauthier le mentionne lui-même: "Le néologisme "sursomption" veut marquer le sens opposé à la subsomption kantienne, qui dans son acception la plus générale suppose qu'on peut soumettre le particulier au général. Le procès inverse de la sursomption "pro-meut" le singulier dans l'universel et propulse le déterminé vers le déterminant (concept) absolu dans le mouvement dialectique qui médiatise toute détermination par le moyen de la double négation» (p. 4-5).

3. Voir "Hegel's Logic from a Logical Point of View", Hegel and the Sciences, R. Cohen et M. W. Wartofsky (dir.), Boston Studies in the Philosophy of Science, vol. 64, Dordrecht, Reidel, I984, p. 303-3 Io, et "Moment cinétique et syllogistique dynamique chez Hegel ", Philosophiques, vol. $32, \mathrm{n}^{\circ} 2,2005$, p. 357-368.

4. Voir par exemple la brève mais bonne introduction de Jean-François Kervégan, Hegel et l'hégélianisme, Paris, PUF, 2005. 
dans le premier chapitre de l'ouvrage, la Science de la logique reçoit un examen dans le chapitre suivant. Comment Gauthier parvient-il ici à dégager une phénoménologie du langage chez Hegel? En retraçant les multiples stations par lesquelles doit passer la conscience avant d'atteindre le savoir absolu. Ainsi, dans l'ordre convenu, le philosophe touchera un mot de la "certitude sensible», de la "perception ", de la «force et [de l']entendement ", de la "vérité de la certitude de soi-même ", de la "maîtrise et [de la] servitude», de la «liberté de la conscience de soi» et de la «raison, [de l'] esprit [et de la] religion" - après quoi il conclura sur le savoir absolu et rappellera l'aspect systématique promis par Hegel dans la préface à la Phénoménologie de l'esprit.

On le devine déjà : suivant le fil des stations rencontrées, il s'agit pour Gauthier d'analyser la place incontournable qu'occupe le langage chez Hegel. Cet examen sera accompli au cœur du développement lui-même, certes, mais aussi et en grande partie dans la conclusion de l'ouvrage. "On peut distinguer quatre phases ou quatre moments du devenir du langage dans la Phénoménologie de l'esprit» (p. 56), suggère l'A. en conclusion. Le premier moment s'exprime dans ce que Hegel affirme sur la certitude sensible. Pour Hegel, «la certitude sensible est contradictoire [car] le ceci, l'ici et le maintenant qu'elle tente de saisir dans leur singularité immédiate se dissolvent dans l'universalité du langage » (p. 56-57). C'est qu'en aucun cas on ne peut parvenir à rendre compte de l'immédiat autrement que par le moyen du langage lui-même, de sorte que l'immédiateté semble ou bien inaccessible ou bien représenter un terme creux. Le second moment du devenir du langage se rapporte pour sa part à l'extériorisation de la conscience. "Sans se manifester ou s'extérioriser, souligne Gauthier, l'Esprit ne pourrait se connaitre, ne pourrait devenir pour soi» (p. 57). Il doit donc forcément y avoir "passage de l'immanence du langage à sa transcendance ou à son objectivation» (p. 57), ce qui implique à la fois une capacité d'identification et de discrimination, le dédoublement de la conscience « en concept et conscience» (p. 58).

Le troisième moment identifié par l'A. consiste dans l'apparition du langage au sein de la culture. "Dans la culture, le langage trouve une demeure; Hegel dit du langage dans la culture qu'il est l'existence concrète (Dasein) de l'Esprit. La culture, c'est le monde de l'esprit objectif ou objectivisé, de l'Äusserung de l'Esprit» (p. 59). Mais le langage ne fait pas que trouver son lieu dans la culture et s'exprimer dans un monde éthique, il se rattache aussi à un "esprit religieux qui accomplit et sursume à la fois le monde objectif» (p. 59). Là se trouve la quatrième phase relative au langage dans la Phénoménologie de l'esprit. Cette phase renvoie à l'idée qu'une fusion doit s'opérer entre l'intériorité et l'extériorité, dans la mesure où le culte, en tant qu'acte religieux, "est la sursomption de la différence entre l'intérieur et l'extérieur; l'individu et le peuple ne font plus qu'un dans l'acte cultuel» (p. 59). 
Avant d'en arriver à l'étude de ces moments charnières, Gauthier avait reproduit, rappelons-le, le trajet général parcouru par la conscience afin d'atteindre le savoir absolu. Le propos qu'il tenait alors était avant tout de nature didactique; mais il s'entourait également de références en tous genres. De Newton à Lacan, en passant par McTaggart, Bosanquet, Josiah Royce, Nagajurna et le bouddhisme zen, Gauthier n'hésite en effet jamais à repérer des lignées ou au contraire des contrastes entre Hegel et moult penseurs. Doublé d'un examen du rôle joué par le langage dans la Science de la logique (p. 6o-62) et d'une analyse logique de la dialectique du maître et de l'esclave (p. I9-22), son commentaire a de quoi rassasier. En va-t-il semblablement du traitement qu'offre Gauthier de la logique interne dans la Science de la logique? Tout à fait, pourrait-on dire, car là encore il «sème à tout vent » et évoque une quantité impressionnante de noms en des analyses ramassées. On notera par exemple les références à Kant, Spinoza, Maître Eckhart, Parménide, Héraclite, Mélissos de Samos, Heidegger, Leibniz, Brouwer, Kronecker, Murti, Tchouang-Tseu et la tradition védique de Brahma (Bhagavad-Gîtâ de la Mahabharata). À ceux qui objecteront à Gauthier que ces références laconiques étourdissent le lecteur plus que ne lui apportent un éclaircissement, le logicien pourrait répondre qu'il fournit là des pistes de réflexion d'autant plus utiles que le philosophe qu'il commente a justement eu pour ambition de «reprend[re] la totalité de la pensée occidentale à son compte» (p. 2).

Résumée en quelques mots, la réflexion développée sur la logique interne hégélienne (ou logique du contenu) prend à peu près les traits suivants. Gauthier fait d'abord remarquer que "si la Phénoménologie de l'esprit accomplit le devenir de la conscience dans le Savoir absolu, son résultat, le concept de science, est l'objet de la Science de la logique qui doit l'exposer selon le contenu» (p. 35). Il laisse entendre ensuite que cette importance accordée au contenu se remarque dans l'ontologie forgée par Hegel et dans la détermination par absence de contenu des concepts d'être pur et de néant pur - tous deux impliqués dans une dialectique où le troisième terme est le devenir. À ce moment, l'A. devient en mesure de conclure qu'il y a bel et bien une logique du contenu chez Hegel, ce qui est renforcé par le fait que Hegel affirme lui-même que l'Idée absolue, vers laquelle il s'agit de converger, «est l'objet et le contenu uniques de la philosophie» (p. 48).

Plutôt que de s'en tenir à ces considérations et d'achever là son ouvrage, Gauthier insère dans les dernières pages quatre appendices de longueur variable qui regroupent des textes en partie déjà publiés ou présentés en conférence $^{5}$. L'appendice A, somme toute bref, s’intitule «La syllogistique

5. Voir «Moment cinétique et syllogistique dynamique chez Hegel », op. cit., et «Logique hégélienne et formalisation ", texte traduit en français par l'auteur et présenté initialement en allemand à un séminaire de Dieter Henrich sur la Wissenschaft der Logik de Hegel à Heidelberg en juin 1966 . 
d'Aristote à Hegel ». Il rapporte avec pédagogie les figures aristotéliciennes du syllogisme et explique que chez Hegel « [1]a tension (Widerstreit) entre les contraires (et subcontraires) se résorbe dans la réconciliation (Versöhnung) du même et de l'autre ou de l'un et du multiple dans une synthèse finale » (p. 68). L'appendice B est quant à lui plus substantiel: il a pour titre «Moment cinétique et syllogistique dynamique chez Hegel ». Distincte de la "syllogistique statique» d'Aristote, la syllogistique dynamique insuffle un élan de vie au concept : à la copule «est » elle substitue la copule «devient »; au lieu de penser la permanence, elle témoigne de l'évolution du savoir et donne lieu à des raisonnements dans lesquels plusieurs concepts «s'échangent le rôle de moyen terme» $(\mathrm{p} .74)^{6}$.

Si cette conception de la syllogistique a été théorisée assez récemment et semble encore bien assumée par l'A. — tout comme l'est d'ailleurs ce qu'il avance sur le "cercle des cercles" au sein de l'appendice D - , Gauthier a maintes réserves à exprimer sur le contenu de son troisième appendice: «Logique hégélienne et formalisation ». Dans une longue et fort instructive note de bas de page, il qualifie en effet d' «enthousiastes» et de «naïves » les prétentions qu'il manifestait dans ce texte écrit en I966. Depuis lors, confesse-t-il, «j'ai sonné le glas sur les entreprises de formalisation de la logique dialectique de Hegel» et "[i]l m'est [...] apparu [...] qu'il est peu utile de formaliser une logique de type hégélien» (p. 95). Geste à la fois honnête et ambitieux, s'il en est, puisque la longue note rédigée par l'A. est autant l'occasion pour lui de revoir ses prétentions que de marquer l'introduction d'un nouveau projet, relié à la syllogistique dynamique de $\mathrm{Hegel}^{7}$, qui "pourrait avoir des extensions et des ramifications du côté de l'intelligence artificielle et de l'informatique» (p. 96).

Certains lecteurs s'étonneront sans doute de ce que le noyau de l'ouvrage totalise au plus 62 pages, auxquelles viennent s'ajouter 35 feuilles d'appendices constitués de travaux déjà parus. D'aucuns interrogeront certainement aussi l'angle sous lequel Hegel est abordé par Gauthier, c'est-àdire un angle «mi-introductif mi-essayistique». Pourtant, et c'est ce sur quoi il importe d'insister ici, ces deux particularités pourront très bien être rangées du côté des vertus de l'ouvrage. Car la série d'appendices construite par Gauthier s'intègre tout naturellement au reste du livre, lequel va droit à l'essentiel, évite de vains détours et atteint son objectif de synthèse par une mise en avant de travaux passés. En donnant un accent mi-introductif mi-

6. Voir aussi Yvon Gauthier, La logique du contenu. Sur la logique interne, Paris, L'Harmattan, 2004, p. I34.

7. Comme le note Gauthier, la logique hégélienne de la sursomption est non booléenne (p. 24-25, 96), car alors que chez Boole " $\neg \mathrm{A}=\mathrm{A}$ », chez Hegel, " $\neg \mathrm{A}=\mathrm{B}>\mathrm{A}$ ». C'est que Hegel considère que la double négation dialectique a cette conséquence particulière de supprimer et à la fois de conserver des moments; de sorte qu'elle entraîne positivement la conscience qui l'effectue à un "niveau supérieur", un niveau qui la rapproche toujours davantage du savoir absolu. 
essayistique à ses écrits, le philosophe a le mérite par ailleurs de permettre à un large lectorat de s'initier à la pensée hégélienne, mais aussi d'amorcer un approfondissement d'une logique singulière. Comment alors qualifier en somme le volume de Gauthier? Sans l'ombre d'un doute en employant les mots de synthèse habile et savante, synthèse qui ouvre la voie à de nombreux travaux logiques et dont l'A. tient à mentionner, en fin d'ouvrage, qu'il veut les «laisse[r] aussi à d'autres» (p. 96).

PIERRE-ALEXANDRE FRADET

Université de Montréal

\begin{abstract}
Claude Ameline, Traité de la volonté, précédé de L'Art de vivre heureux (attr. à C. Ameline), édition, introduction et notes de Sébastien Charles, Paris, Vrin, coll. «Textes cartésiens en langue française », 2009, $294 \mathrm{p}$.
\end{abstract}

Espérons que la série des "Textes cartésiens en langue française ", dirigée par Denis Moreau et éditée dans la collection «Bibliothèque des textes philosophiques» chez Vrin, facilitera des recherches précises sur ce qu'on nomme parfois trop vaguement l'héritage cartésien. Des textes d'Arnaud, de Cordemoy, de Desgabets, de La Forge, et de bien d'autres cartésiens sont à paraître. L'intérêt de rééditer ces auteurs qui ont écrit dans le sillage de Descartes est avant tout pratique. Les textes de la plupart de ces écrivains de la seconde moitié du XVII ${ }^{\mathrm{e}}$ siècle sont désormais soit indisponibles soit accessibles, mais dans des éditions peu conviviales. D'où l'effort de cette série qui cherche à éviter que tombe dans un oubli complet un pan du patrimoine philosophique français. C'est donc dans cette optique éditoriale que nous retrouvons la réédition du Traité de la volonté ( 1684 ) de l'oratorien Claude Ameline, précédé de L'Art de vivre heureux (I667, I690) qui lui fut vraisemblablement faussement attribué ( Pseudo-Ameline» sera alors tout indiqué pour nommer l'auteur anonyme de ce traité). L'introduction et les notes de Sébastien Charles tentent avec intelligence de jeter quelque lumière sur cette attribution douteuse. Toutefois, d'entrée de jeu, S. Charles nous avise que son choix éditorial ne se réduit pas à prouver si Ameline est bel et bien l'auteur de L'Art de vivre heureux:

Mais au fond, la question de la paternité de l'Art de vivre heureux n'est pas l'essentiel. Ce qui importe, c'est de voir que ces deux traités sont intéressants indépendamment de l'attribution qu'on peut en faire, parce qu'ils rendent compte à eux deux de l'état d'esprit propre à la philosophie chrétienne de la fin du XvII ${ }^{\mathrm{e}}$ siècle, marquée plus ou moins profondément par l'héritage cartésien (p. I 8).

L'interrogation qui anime ces deux traités s'attache à déterminer la possibilité de parvenir au bonheur malgré la concupiscence dans laquelle baigne l'humanité depuis la chute d'Adam. Le problème capital s'avère donc 